\title{
La technique des prescriptions sacrificielles dans la géomancie bambara (région de Ségou, Mali)
}

The technique of sacrificial prescriptions in Bambara geomancy (Segou area, Mali)

Christian Bertaux

\section{OpenEdition}

\section{Journals}

Édition électronique

URL : https://journals.openedition.org/span/540

DOI : $10.4000 /$ span. 540

ISSN : 2268-1558

Éditeur

École pratique des hautes études. Sciences humaines

Édition imprimée

Date de publication : 1 avril 1984

Pagination : 117-130

ISSN : 0294-7080

Référence électronique

Christian Bertaux, "La technique des prescriptions sacrificielles dans la géomancie bambara (région de Ségou, Mali) », Systèmes de pensée en Afrique noire [En ligne], 6 | 1984, mis en ligne le 05 juin 2013, consulté le 28 juin 2022. URL : http://journals.openedition.org/span/540 ; DOI : https://doi.org/ $10.4000 /$ span. 540 
L A T E C H N I Q U E D E S P R E S C R I P T I O N S
S A C R I F I C I E L L E S
D A N S
L A
G E O M A N C I E

B A M B A R A ( R E G I O N

D E

$\mathrm{S} E \mathrm{E} O \mathrm{U}$,

M A L I ) 1

par

Christian Bertaux

Parmi les nombreuses géomancies à seize figures utilisées en Afrique noire, la géomancie bambara (tiènda, tinyèda, bugurida, respectivement "sable", "vérité", "poussière") ${ }^{2}$ se caractérise par sa façon de faire sortir (bò) les calligrammes divinatoires, par sa manière de nommer les lieux (tiènsow, litt. "maisons du sable") où viennent se loger les figures de géomancie (tièndew, "enfants du sable", ou tinyèdenw, "enfants de la vérité"), par son interprétation des paroles (kumaw) que produit la répétition des figures sur le tableau géomantique (tiènfan, litt. "l'oeuf du sable"), par sa construction de trois tableaux

1. En pays bambara, la géomancie, plus prestigieuse que la divination par les cauris, concerne à la fois des problèmes individuels (santé, argent, ennemis) et institutionnels (fêtes des sociétés d'initiation, sorties de masques, etc.). Introduite au Mali à une époque sans doute très ancienne (Mansa Souleyman -- 1341-1360--, que Ibn Batouta a rencontré lors de son voyage en 1352 dans l'Empire du Mali, est le nom d'une des figures de cette géomancie), elle apparaît aujourd'hui comme techniquement différente des géomancies du Soudan (Darfur), de Haute Volta (Gourmantché), de Mauritanie (Nouadhibou) et de la géomancie arabe des colporteurs.

2. L'opérateur da qu'on retrouve dans toutes ces appelations est un lexème très complexe qui signie "étendre", "poser", "coucher", etc. Le géomancien (tièndala) est celui qui "étend" (da) le sable (tièntien ou cèncèn), qui "étale" la dimension créatrice de la parole. C'est pour désigner cette "longueur" dans l'acte de production très soigné des figures de géomancie sur le sable (seize algoritmes composés de quatre traits pairs ou impairs) que nous utilisons 1'expression "ca1ligramme divinatoire" . Cette même technique bambara a d'autres noms, tous liés à 1 'opérateur da : tinyèda. (de tinyè, "vérité"), bugurida (de buguri, "poussière"), trabuda (de trabu, sable). 
dérivés et emboîtés les uns dans les autres (sanw, 1itt. "années") et, enfin, par ses codes sacrificiels (saraka bò, litt. "sortir les offrandes").

Lorsque le devin (tièndola) fait sortir de la bouche de la terre des figures qui se répètent, il appréhende un "bavardage" (kuma caaman, litt. "parole nombreuse") générateur d'événements. Or ce bavardage calligraphique des enfants du sable renvoie à celui que produisent les puissances oraculaires, mâ̂tres mystiques de la brousse, à savoir les huit personnes (mogo segin) et les huit djinns (jinè segin) ${ }^{3}$, invoqués avant toute consultation par le devin. Le géomancien bambara suit dans sa gestuelle -- selon un espace orienté de la brousse vers le village et de 1'est vers 1'ouest -- le flux des événements engendrés par des signes. C'est parce qu'il dégage sur le sable ces signes issus de la parole des puissances oraculaires qu'il peut "voir" (flèzi) ${ }^{4}$ les problèmes et modifier la production des événements.

L'emboîtement des trois tableaux géomantiques se déroule dans la pratique du devin comme une parole créatrice (kuma) qui, en roulant la terre $^{5}$, produit et développe les récoltes d'une suite d'années (sanw).

3. Les huit personnes et les huit djinns sont systématiquement invoqués dans la prière que fait le devin sur une prise de sable avant de faire apparaître les figures qui seront utilisées pour construire le tableau divinatoire. Cette prière utilise une formule stéréotypée (toujours dans cet ordre : "personne huit et djinn huit") qui fait référénce à deux grandes catégories oraculaires : les figures ayant un nombre de traits pair (mògòw, "personnes") et les figures ayant un nombre de traits impair (jinèw, "génies"). Ces êtres géomantiques, à 1'état de calligramme sur le tableau divinatoire, peuvent se percevoir en brousse sous la forme d'êtres fantastiques : nains, géants, personnes humaines vivant en brousse.

Selon $Y$. Cissé, les huit figures géomantiques appelées "personnes" représentent les huit premiers ancêtres mythiques de 1 'humanité liés à Faro (divinité de $1^{\prime}$ eau), alors que les huit djinns sont des êtres issus d'une première création ratée, associée à Mousso Koroni (personnalité mythique liée à la catégorie du sec, à la souillure, à la démence, à 1 'errance...).

4. La géomancie bambara est pensée, au même titre que les autres techniques divinatoires (cauris, calebasses, etc.) dans la catégorie du "voir" (flèli). Elle est ce qui "fait voir" (flèlikè) et le géomancien "celui qui fait voir" (flèlikèza).

5. La production des calligrammes sur le sable suit le cheminement de la parole (kuma taama) désïgné dans la gestuelle du géomancien. La référence à 1 'enroulement et au déroulement de la parole est liée à 1 'image du bousier (bo kolonkolon, litt. "celui qui roule les excréments"). 
En regardant le premier tableau, le devin dévoila la première année (san fözò) et appréhende une parole à son commencement (kuma daminè), à I'état de "suspension". En regardant le second tableau ou la seconde année (can fizana), il développe la parole en son milieu (kuma cèmancè) pour 1'achever (kuma Zaban) avec le troisième tableau ou la troisième année (san sabana). Dans la mesure où les prescriptions sacrificielles sont une autre manière d'achever le déroulement de la parole, le devin, dans son effort de retravailler à sa source 1 'engendrement des événements, ne peut les établir qu'à partir du premier tableau, lorsque les paroles sont à leur commencement, à 1'état de suspension. La production des deuxième et troisième tableaux vient, en revanche, offrix une parole achevée et comblée qui termine la consultation.

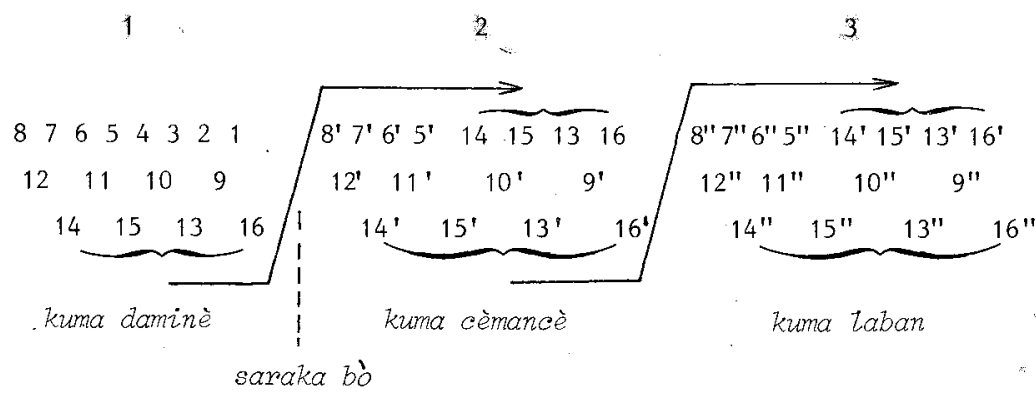

Fig. 1

Les trois étapes de la parole (kuma) dans le déroulement des tableauz géomantiques ${ }^{6}$

A chaque figure de géomancie correspondent des demandes sacrificielles, des "envies" (myèw). Ainsi, musa (2222) aime le beurre de karité, ali bayaro 2212) le lait, muhunkoro. (2211) les vieux habits...

Nongonforokoro (1itt. "vieux champ de fumier"), 1'un des maîtres mythiques de la géomancie bambara (cf. infra p. 129) est parfois associé au bousier.

6. Les quatre dernières figures du premier tableau deviennent les quatre premières du second tableau et les quatre dernières figures de celui-ci sont renvoyées au début pour faire le troisième tableau.

Les tableaux se superposent dans "1'oeuf de sable". Les nombres situent ici les seize maisons et la circulation minimale des figures pour construire les trois tableaux. 
Les offrandes (saraka) peuvent être constituées par les matériaux suivants : colas, tissus, sel, tabac, racines, feuilles médicinales, poudre de fusil, habits, écorce, eau, poulets, moutons, boucs, pintades, oeufs, maîs, lait frais, insectes, margouillats, crapauds... Mais le terme générique saraka bò désigne également de simples procédures d'évitement, plus importantes quelquefois que les offrandes elles-mêmes. lléanmoins, les codes sacrificiels les plus élaborés impliquent obligatoirement diverses variétés de colas (worow) et de poulets (shèw) :

-- cola bianche, cola rouge, cola à tête de cheval, cola qui rigole, cola ni rouge ni blanche, petite cola, vieille cola, cola qui a la forme d'une noix de karité...

-- poulet blanc, poulet noir, poulet vautour, poulet mouton, poulet tourterelle, poulet noir à poitrine blanche, poulet de toutes les couleurs, poulet qui n'a pas encore crié...

Il s'agit là de matériaux concrets, d'usage 'quotidien, qui permettent de retravailler -- selon telle ou telle figure et en fonction de telle ou telle maison précisant un problème -- des flux oraculaires.

Les relations qu'il y a entre la sortie (bó) des figures de géomancie sur le sable et la sortie des offrandes sacrificielles (saraka). n'obéīssent pas à un savoir taxinomique rigide. Les correspondances changent d'un devin à $l^{\prime}$ 'autre et même d'une consultation à 1 'autre chez les même devin. Plusieurs solutions sont souvent possibles. Les devins répugnent à produire des systèmes complets et à parler de manière spéculative des entités oraculaires.

\section{Crône de boeuf et huit colas}

Lorsque le géomancien Baboli Traoré, chef de la société du Komo (société d'initiation) du village de Tio (à $50 \mathrm{~km}$ de. Ségou), rencontre sur son tableau divinatoire quatre fois quatre traits pairs, il doit faire un sacrifice, c'est à dire "sortir une offrande" (saraka bò). La parole (kuma) déclenchée par la sortie de ces traits répétant quatre fois la figure musa (2222) est tellement volubile qu'il va falloir 1'ôter, la contrôler, la "sortir"7.

7. Un tableau géomantique est constitué par seize figures qui sont 


\section{$\begin{array}{llll}\| 1 & 11 & 11 & 11 \\ 11 & 11 & 11 & 11 \\ 11 & 11 & 11 & 11 \\ \text { II } & 11 & 11 & 11\end{array}$}

Fig. 2

musa kuma caaman

(le bavardage extrêne de misa)

Pour Baboli Traoré, cet acte de "sortir" devra tenir compte du contexte. Si la production des quatre msa s'est faite dans une situation de malheur extrême, il faudra aller chercher le crâne d'un boeuf mort. Briser ce crâne. L'enduire, à I'intérieur, de beurre de karité pour satisfaire musa en brousse. Ramasser du sable divinatoire où musa a été calligraphié. Faire une incantation (kizisi) sur cette prise de sable-msa en lui expliquant qu'il y a du malheur dans la communaté; que cela doit se terminer, qu'il doit aller bavarder ailleurs. Mettre le sable chargé du calligramme de musa et des paroles incantatoires dans le crâne enduit de karité. Emporter le tọut sur une termitière et 1'y abandonner.

Baboli Traoré passe, dans son travail sacrificiel, du généré (musa cal1igrame) au générant (musa en brousse). I1 sort et abandonne 1 'engendrement sémiologique tout en essayant de satisfaire musa logé dans le crâne enduit de beurre de karité.

logées en seize lieux sur le sable divinatoire qu'on appelle des maisons (sow). Il y a, lors de la construction du tableau, toujours au moins une répétition des figures. C'est sur le repérage des répétitions que s'organisent principalement, en pays bambara, la notion de "bavardage" des figures et l'identification des problèmes. Au cas où quatre musa (4 fois. 2222) "sortent" dès le départ, le bavardage est extrême, car quatre musa produisent seize musa sur le tableau divinatoire. Un tel bavardage est dangereux pour la communauté, indépendamment de la sémantique de la figure (2222). Celle-ci a peu d'importance en pays bambara (est même parfois inconnue des devins). Au niveau de la pratique arabe de la géomancie (différente de la pratique bambara), cette figure est traditionnellement associée à jemac, la "communauté". La géomancie bambara utilise, à la différence des géonancies européenne et gourmantché, un ordre associant les figures aux maisons (qu'on trouve également dans les oeuvres de Ez Zénati). janfa almani (1121) est ainsi maître de la maison de l'âme (ni), alimangusi (2221) de la tombe (bogo), musa (2222) de la "fin des choses" (Zaban), etc. 
Lorsque la sortie des quatre calligrammes de musa s'est faite dans une situation d'extrême bonheur, il faut consolider cette chance en suivant une autre procédure. On doit aller chercher huit colas blanches (un jeudi). Expliquer à musa qu'il doit consolider la chance en évitant les effets que risquent de produire autant de répétitions de lui-même. Après 1 'avoir remercié, manger une cola blanche le jour de l'incantation et répéter cet acte pendant huit jours, une cola par jour. Ici, l'engendrement sémiologique produit par msa n'est pas évacué. Retravaillée, la parole calligraphique est réintroduite dans la communauté par l'acte de consommation.

\section{Un bouc tacheté de noir et de blane}

Mamadou Diabaté, un jeune devin réputé de Ségou, un devin soma ${ }^{8}$, attentif à la prolifération des paroles que déclenche la sortie quatre fois répétée du calligramme de musa, va faire également un sacrifice (saraka bò), mais sans tenir compte du contexte (bonheur ou malheur). I1 s'agit d"éviter de laisser ces calligrammes sur le sol". La communauté serait prise de discussions incessantes, indice certain de graves problèmes. Il est donc nécessaire de sortir les calligrammes en effectuant un saraka bò.

On cherche un bouc tacheté de noir et de blanc (nyami bakoro). On dessine quatre fois musa sur le sol près de la maison du devin. On ramasse le sable calligraphié et on fait une incantation (kizisi) exliquant à msa qu'il doit aller ailleurs. On met le sable calligraphié et chargé de paroles dans la bouche du bouc vivant. On 1'égorge alors sur le tableau divinatoire, sur le sol où était calligraphié musa. On mange ensuite le bouc, le foie et le coeur revenant au devin.

8. Mamadou Diabaté est un devin soma d'une quarantaine d'années. Comme tout devin soma, il a été directement initié par les djinns. Perdu en brousse lorsqu'il était enfant, il y a été nourri et protégé par eux. Depuis, il pratique quotidiennement la géomancie dans les environ de Ségou. C'est un fils de griot (jeli). Il conseille hommes et femmes, musulmans et paīens, sur différents problèmes d'ordre quotidien (métier, amour, argent, santé, voyages, naissances, etc.). Outre sa pratique de la géomancie, il prépare des amulettes (sèbènw) et des fétiches (buliw).

Les devins Traoré sont, par contre, des agriculteurs. Ce sont des horonw (des hommes libres, non castés), qui se font un point d'honneur de "ne pas gagner de l'argent sur le dos de la terre". 
De musa sur le sable (partie terminale de I'engendrement sémiologique) à musa en brousse (à l'origine de I'engendrement sémiologique), le rapport de transformation établi par ce dernier rite n'est pas le même que dans le cas précédent. Le corps du bouc vient en quelque sorte s'ajuster sur le sable divinatoire (sa bouche étant mise en correspondance avec celle du tableau géomantique). Il absorbe les calligrammes dangereux de musa et les élimine de la communauté villageoise. Pour Baboli Traoré comme pour Mamadou Diabaté, 1a répétition de musa comporte donc un risque très important et, dans les deux cas, il ne faut pas le laisser à terre. Baboli Traoré 1 'enferme dans un crâne de boeuf et le renvoie à la limite du monde cultivé et de la brousse (marquée par une termitière). Mamadou Diabaté utilise le sacrifice d'un bouc pour bloquer 1 'événement en faisant disparâ̂tre dans son corps les calligrammes divinatoires. Les deux devins -- selon leurs façons propres -- tendent à retravailler la génération des événements dans la génération des calligrammes. C'est ce travail qui est une des grandes caractéristiques de la géomancie bambara ${ }^{9}$.

\section{Utilisation d'un épiphyte (ficus iteophylla)}

Baboli Traoré peut recourir aussi à des rites faisant intervenir des arbres pour retravailler certaines circulations des figures de géomancie.

Lorsqu'il y a une répétition de la figure janfa almani (1121) de la maison 1 (1a maison de 1'âme, $n i$ ) à la maison 5 (1a maison des enfants, denw), il peut bloquer 1 'aspect néfaste de cette circulation 1-5 en utilisant un jatigifaga (1itt. "le tueur de son logeur") en train d'étrangler un baobab. Il s'agit d'un parasite (ficus iteophyzza) qui peut devenir aussi immense qu'un baobab et le détruire.

Le rite consiste à offrir (saraka bò) à janfa almani la "trahison

9. L'approfondissement de ce point impliquerait une étude plus complète de la géomancie bambara et de ses diverses variantes en milieu urbain et en brousse. Nous cherchons seulement ici à signaler que, parmi toutes les techniques géomantiques africaines, il y a une façon proprement bambara d'ajuster le registre des prescriptions sacrificielles dans le registre des paroles oraculaires dévoilées par 1 'engendrement calligraphique. Le choix des matériaux que nous avons fait dans cet article répond au souci d'éclairer cette articulation propre à la technique du tièndaza. 
de l'enfant" qu'il tend à réclamer dans la passe 1-5 (janfa veut dire trahison). On doit pour cela aller en brousse $\left(\mathrm{G}^{-1}\right)$ pour "sortir" le flux calligraphique $(G)$ de la communauté. Dessiner le calligramme de janfa almani sur 1'écorce du jatigifaga. Faire une incantation (kilisi) pour que janfa almani se satisfasse de ce mode de trahison dans le "monde des enfants" (une trahison d'"arbre-enfant").

1 ,

(Y)

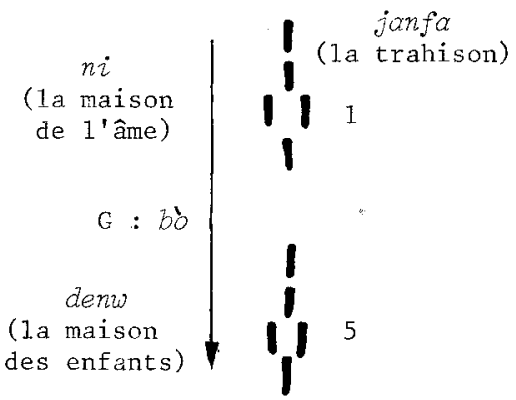

$(\mathrm{X})$
2 .

(Y)

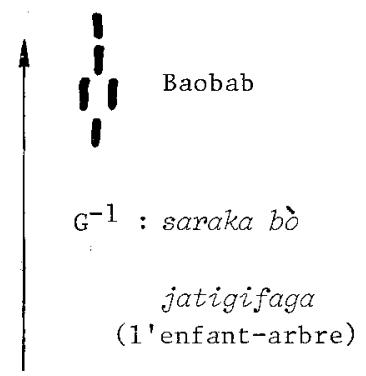

$(\mathrm{X})$

Fig. 3

Flux sémiologique $G$ et contre-Elux $G^{-1}$

( 1 'enfant-arbre, en tuant son logeur, va détourner $I^{\prime}$ avènement de la trahison de la maison des enfants)

La suite du rite comporte la consommation au village de petites branches cuites de jatigifaga. L'enjeu de toute l'opération est, d'une part, de renvoyer la circulation du calligramme de la maison 5 à la maison 1 (come le fait le jatigifaga qui se loge sur le baobab ${ }^{10}$ ), d'autre part, de réintroduire cette circulation (qui pourra être utilisée) dans la communauté par la consommation des branches du ficus. La passe 1-5 est donc conservée (1e lien de 1'âme aux enfants), mais débarassée de la trahison qui suit janfa almani.

10. Il y a un jeu de mot entre chemin (sira) et baobab (nsira ou sir $\alpha$ ). Le baobab vient ici enfouir le cheminement calligraphique. I1 sert souvent de vraie "cache" à fétiches. Il bloque les mauvaises forces et peut servir de tombe. 
4. Utilisation de cinq cauris enterrés sous un arbre

Quand on veut nuire à quelqu'un et que le tableau géomantique fait apparaître une circulation du type précédent (une passation de 1 à 5), on peut tenter d'inverser le flux et de le bloquer au pied d'un arbre. Pour cela, Mamadou Diabaté va chercher cinq cauris (le nombre 5 caractérisant janfa almani, maître de la maison de l'âme). Il porte ces cauris (non taillés, contrairement à ceux qui servent de monnaie) en brousse ; il coupe une petite racine du côté est d'un arbre et il met les cinq cauris à sa place (voir figure ci-dessous). Il fait ensuite une incantation (kilisi) sur eux (c'est-à-dire sur janfa almanì) ; il replace la racine en sens inverse (c'est-à-dire après lui avoir fait subir une rotation de $180^{\circ}$ ) en la posant sur les cauris ; il recouvre le tout de terre.
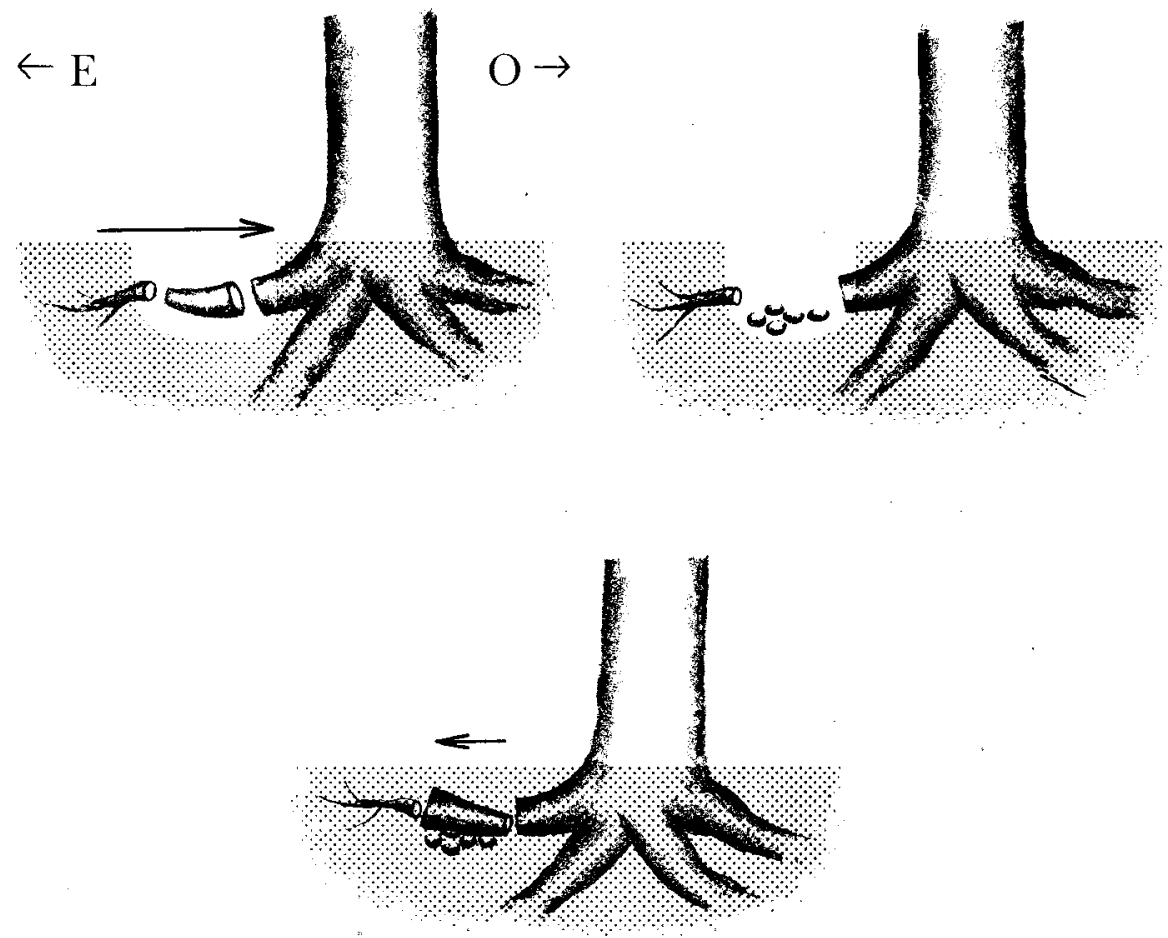

Fig. 4

La racine aux cauris 
Ce qui importe dans cette opération, c'est de produire un contreflux en nourissant janfa almani par une racine mise à l'envers par rapport à la circulation normale de la sève. L'ennemi va dépérir, car sa "maison de l'âme" (maison 1) n'est plus alimentée par la parole de janfa atmani, bloquée sous 1 'arbre.

\section{Procédures particulières liées au mấtre de l'âme}

La figure qui gouverne la maison de 1 'âme $(n i)$, janfa almani, est un djinn, un nain fantastique qu'on peut rencontrer en brousse, à la limite du village. Tantôt habillé, tantôt dénudé, il se caractérise par la trahison (janfa). Lorsqu'on le rencontre, on doit faire une incantation (kilisi) pour ne pas devenir fou ${ }^{11}$.

Il ne faut jamais offrir un sacrifice sanglant à janfa almani lorsqu'il est seul dans la maison de l'âme (maison 1). Cela provoquerait la mort du consultant ${ }^{12}$. Les prescriptions sacrificielles impliquées par janfa almani doivent suivre une sémantique de la trahison et tout un jeu de relation indirectes. Donnons un exemple.

Lorsque janfa almani va dans les maisons 4-5-14 (le père, les enfants et la fortune), on doit faire un saraka bò. Pour le chef'du Komo du village de Kirango, Karamango Traoré, on doit essayer d'obtenir auprès d'un vendeur deux colas blanches sans donner de 1'argent en échange (on dira notamment au vendeur qu'on 1'a oublié). On ramène les colas chez soi, on fait sur elles une incantation, on les mange et c'est alors seulement qu'on peut payer le vendeur.

Cet exemple montre que la trahison liée à janfa almani peut aussi être bonne et qu'on ne peut avoir la fortune (maison 14) sans "déviation". L'acte de. "sortir 1'offrande" passe par cette déviation, plus importante encore, dans la relation à janfa almani, que 1'offrande

1. "On ramasse sur le sol quatre pierres de petite taille. On fait une incantation sur ces quatre pierres. On jette ensulte les pierres une à une. Une pierre en avant. Une pierre en arrière. Une pierre à gauche. Une pierre à droite. Le djinn disparaît alors." (Mamadou Diabaté, Ségou, 1980).

12. On peut se demander si l'absence des outils sacrificiels à un lieu de jonction important du consultant n'est pas une propriêté générale de la divination. Dans 1a tradition gêomantique de Cattan (XVIe siècle), un thème de géomancie ne doit pas être inferprété lorsqu'il y a en maison 1 cauda draconis (le couteau) ou mibeus (le sang). 
elle-même. Un simple détour (quelques pas hors du groupe lors de l'arrêt d'un taxi de brousse) peut rendre propice un voyage lorsque la consultation fait apparaître janfa almani en maison 9 (la maison des voyages).

6. Le retraitement de la parole de alimangusi par un poulet noir

Nous avons vu que les prescriptions du type saraka bò tendent à renvoyer aux limites de la brousse, à la source de l'engendrement de la réalité par les signes, certains effets de ces signes. La "sortie" des offrandes bloque, étaye, déporte ou filtre celle des calligrammes.

Précisons cela par un nouvel exemple. Soit le déplacement 2-10 d'un djinn, alimangusi, maître de la mort :

1.

Maison 2 : maison de la chance

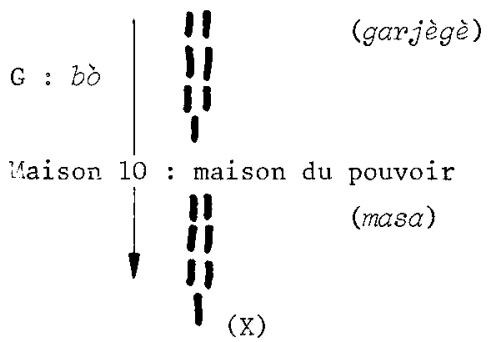

2 .

En brousse

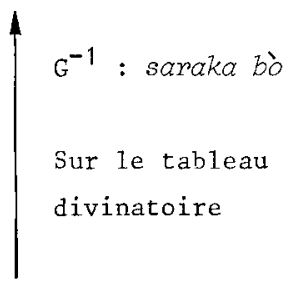

(x)

Fig. 5

Prescription sacrificielle liée

au développement de la parole (kuma) de alimangusi passant de la maison 2 à la maison 10

La "passe" 2-10 de alimangusi (2221) indique qu'il va y avoir de 1a chance (garjègè) dans le pouvoir (masa), mais que cette chance risque de se produire d'une manière criminelle, car elle est gouvernée par le maître de la mort. Que faire ? La prescription sacrificielle va chercher à "filtrer" cette passe, à enlever la dimension du crime tout en conservant la dimension utilisable "argent dans le pouvoir". Pour cela, les devins Mamadou Diabaté et Karamango Traoré indiquent la procédure suivante :

-- aller chercher un poulet noir (offrande aimée par alimangusi pour 1a 
"noirceur" de ces crimes;

-- dessiner alimangusi sur le sable divinatoire effacé de son thème ;

-- poser les pattes du poulet sur le sable calligraphié (afin que alimangusi-calligramme suive alimangusi-poulet) ;

-- faire une invocation en disant à alimangusi qu'il doit partir ;

-- aller en brousse à l'est d'un arbre (la source du flux sémiologique étant située à l'est) ;

-- faire sept fois une invocation à alimangusi (ce qui remplace 1'inscription du calligramme, 7 étant le chiffre de ce djinn) ;

-- expliquer à alimangusi (calligramme et poulet noir) qu'il ne faut pas qu'il y ait crime et qu'il doit se satisfaire du "noir" de ce poulet ;

-- écraser le poulet noir contre la partie est de l'arbre (afin que ce dernier bloque I'engendrement sémiologique);

-- ramasser des bouts d'écorce portant des traces de sang et des plumes

à 1 'est de 1 'arbre (où alimangusi a fait cible) ;

-- rentrer chez soi de 1 'est vers l'ouest (selon le flux sémiologique);

-- faire une décoction avec les bouts d'écorce qu'on a ramenés ;

-- se laver pendant les sept jours qui suivent avec cette décoction mêlée à l'eau du bain.

Le bain ( 1 'acte de consommation aurait le même effet) vient inclure I'intéressé dans le flux sémiologique retravaillé par le sacrifice. Si le devin voulait simplement éliminer la passe 2-10 de alimangusi, il se contenterait de 1 ' abandonner (sur une termitière, par exemple).

\section{La légende de Djitoumou Bala et de Nongonforokoro}

L'articulation entre l'engendrement sémiologique de la réalité que nous avons noté $G$ et les prescriptions sacrificielles (saraka bò) qui cherchent à repousser ou à faire muter G par des "réalités-signes" (sarakatiw -- offrandes, gestes, paroles, déplacements, abandons, évitements, etc.) est une caractéristique fondamentale de la géomancie bambara. Elle apparaît clairement dans 1'invocation que tous les devins font à la terre avant la consultation. Après avoir étalé le sable divinatoire et tracé dessus un calligramme "aute1" (une des seize figures, liée à l'initiation du devin et à 1 'heure de la consultation), le géomancien fait une invocation (kilisi) en portant le sable de ce 
calligramme à sa bouche. Dans cette invocation ${ }^{13}$, il commence par une parole d'évacuation ( $t u$ bisibizay), puis il nomme les deux mâttres mythiques de la géomancie bambara, Djitoumou Bala et Nongonforokoro. Il peut alors faire appel à l'entité du calligramme "autel", puis aux maîtres des figures de géomancie, les huit personnes (mògo segin) et les huit djinns (jinè segin). Ainsi, l'ajustement à la source de l'engendrement sémiologique se fait sous la protection du calligramme "autel" et des deux grands mâtres mythiques de la divination. L'articulation $G / G^{-1}$ se retrouve dans la relation que ces derniers entretiennent entre eux. La technique bambara des prescriptions sacrificielles est plus particulièrement liée à Nongonforokoro. Chacun connaît son histoire. Voici ce que Mamadou Lamine Traoré écrit à ce sujet :

"I1 s'agit de 1'histoire de Djitoumou Bala (1égende ou mythe ?), un grand mâ̂tre géomancien, connu dans tout le pays. Après une longue vie sans jamais être tombé malade, un jour il se coucha et ne se leva plus : une étrange maladie l'avait terrassé. Tous les disciples s'essayèrent à le tirer de là, tous les mâtres, amis ou rivaux. Nul ne le put. Et pourtant il n'arrivait plus à se lever, il ne se réveillait plus (kunubali), mais il ne mourait pas non plus (sabali). Immortel et inéveillé. Un jour que tous les géomanciens de la région étaient réunis et demandaient conseil à la terre, voilà que passa un petit garçon qui vint effacer du pied droit toutes les figures de géomancie et dit aux vieux sages : "Allez chercher la plus grosse branche d'un kunjè (guiera senegalensis) que vous puissiez trouver, déposez-la sur une natte dans la cour de votre mâ̂tre et couvrez-1a d'une couverture qui n'a jamais servi. Les vieux firent comme I'enfant leur avait dit et quand ils eurent fini, ils furent stupéfaits de constater que Bala, le grand maître de la vérité avait disparu. (...) Le petit garçon, Nongonforokoro, le remplaça à la tête des géomanciens du pays."14

13. Les devins bambara qui ont pu entrer en contact avec 1'islam insistent sur le fait que les paroles produites dans la géomancie bambara ne sont pas des prières. S'il s'agissait de prières au sens théologique, cela entraînerait directement la mort du devin en quelque sorte sacrifié aux entités oraculaires priées. Il y a donc une différence essentielle entre les paroles géomantiques bambara et les prières théologiques, entre les invocations (ou les incantations) et les paroles de la prière musulmane. L'invoquant doit se "ramparder" face aux puissances dangereuses qu'il réveille. Les offrandes d'eau faites sur le sable divinatoire permettent également au géomancien de conserver une distance vis-à-vis d'un sable desséchant (représenté comme antérieur au règne des terres cultivées) qui pourrait emporter sa forme, son ja ("double").

14. Mamadou Lamine Traoré, Vers une pensée originelle africaine. Exposé géomantique. Critique de la négritude et du consciencisme (Thèse de doctorat de troisième cycle, Université de Pairs IV, Année 1978-79). 
Djitoumou Bala et Nongonforokoro représentent deux générations de devins en même temps que deux modes d'articulation dans le rapport du géomancien à la parole de la terre. L'un est le mâ̂tre des figures de vérité qui viennent parler de l'engendrement du monde (G). L'autre est le mâtre de la mise en scène des prescriptions sacrificièlles qui permettent de retravailler (G-1) avec des réalités-signes (sarakatiw) 1 'engendrement $G$ dévoilé sur le sable.

A 1a première génération, il s'est produit une sorte d'impasse. Tout en devenant très vieux et comme absent à ce monde (inéveillé, kunubali), Djitoumou Bala, protégé par sa mâttrise des enfants du sable qui 1'associaient aux entités oraculaires de la brousse, était incapable de mourir (immortel, sabali). Nongonforokoro, un jeune garçon venu d'ailleurs, a pu modifier la situation en faisant muter la relation du mâttre au flux sémiologique G. Avec des sarakatiw (des réalités-signes tels le mouvement du pied effaçant ie sable, la branche de kunjè, la couverture qui n'a jamais servi et qui fait référence à un rituel de deuil...), il a su limiter la puissance de ce flux. En effaçant les calligrammes laissés par Djitoumou Bala, il a fait, tel le bousier, rouler la terre et passer le dessous sur le dessus. En amenant dans une cour du village une branche de kunjè (litt. "tête blanche") prélevée en brousse et en recouvrant celle-ci d'une couverture comme on le fait pour un cadavre, il a fait mourir son aîné.

Cette façon de renvoyer Djitoumou Bala dans 1'espace d'immortalité des entités divinatoires en limitant les effets de ces dernières au niveau du village peut être considérée comme le premier acte de "sortir une offrande" (saraka bo) des géomanciens bambara. Toutes les prescriptions divinatoires semblent suivre cette technique. 


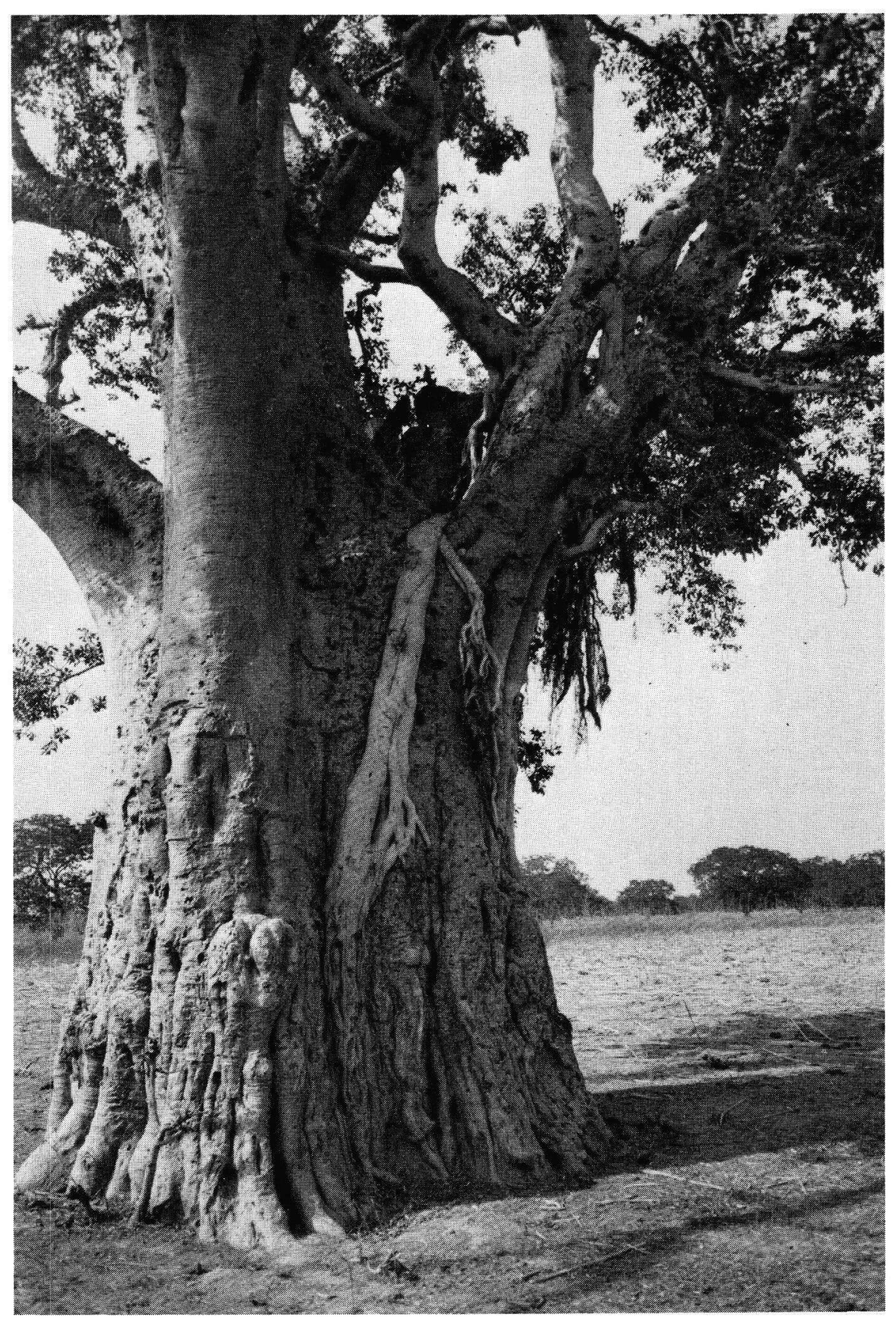

jatigifaga étranglant un baobab 